\section{Medicina Tradicional e Complementar no Brasil: inserção no Sistema Único de Saúde e integração com a atenção primária}

\author{
Traditional and Complementary Medicine in \\ Brazil: inclusion in the Brazilian Unified National \\ Health System and integration with primary care
}

\author{
Medicina Tradicional y Complementaria en Brasil: \\ inserción en el Sistema Único de Salud e \\ integración con la atención primaria
}

Islandia Maria Carvalho de Sousa 1

Charles Dalcanale Tesser 2,3

doi: 10.1590/0102-311X00150215

\section{Resumo}

Este estudo objetivou analisar a inserção da Medicina Tradicional e Complementar no SUS e sua integração com a atenção primária à saúde. Realizouse pesquisa qualitativa baseada em dados institucionais, artigos indexados e estudos de casos em municípios brasileiros selecionados: Campinas (São Paulo), Florianópolis (Santa Catarina), Recife (Pernambuco), Rio de Janeiro e São Paulo. A análise foi realizada na perspectiva da inserção da Medicina Tradicional e Complementar na rede assistencial e sua integração com a atenção primária à saúde, por meio das seguintes dimensões: presença da Medicina Tradicional e Complementar na agenda municipal; posição nos serviços; modo de acesso; praticantes; tipos de práticas; perfil da demanda; potencial de expansão no SUS. Foram identificados e caracterizados quatro tipos de inserção e integração da Medicina Tradicional e Complementar, associados ou não: Tipo 1 - na atenção primária à saúde via profissionais das equipes de saúde da família - Integrada; Tipo 2 - na atenção primária à saúde via profissionais de exercício exclusivo - Justaposta; Tipo 3 - na atenção primária à saúde via equipes matriciais - Matriciada; Tipo 4 - em serviços especializados - Sem integração. A combinação dos tipos 1 e 3 foi considerada uma diretriz potencial para a expansão da Medicina Tradicional e Complementar no SUS e pode orientar o crescimento e sua integração na atenção primária à saúde. A crescente presença da Medicina Tradicional e Complementar no SUS demanda pensar estrategicamente sua expansão, e não devem ser desperdiçadas as experiências existentes.

Medicina Tradicional; Terapias Complementares; Atenção Primária à Saúde; Sistema Único de Saúde

\author{
Correspondência \\ I. M. C. Sousa \\ Centro de Pesquisas Aggeu Magalhães, Fundação \\ Oswaldo Cruz. \\ Av. Professor Moraes Rego s/n, Recife, PE 50670-420, Brasil. \\ islandia@cpqam.fiocruz.br \\ 1 Centro de Pesquisas Aggeu Magalhães, Fundação Oswaldo \\ Cruz, Recife, Brasil. \\ 2 Centro de Ciências da Saúde, Universidade Federal de Santa \\ Catarina, Florianópolis, Brasil. \\ 3 Centro de Estudos Sociais, Universidade de Coimbra, Coimbra, \\ Portugal.
}




\section{Introdução}

O uso da Medicina Tradicional e Complementar tem aumentado nas últimas décadas nos sistemas de saúde 1,2,3. O que a literatura científica biomédica denomina Medicinas Alternativas e Complementares (Complementary and Alternative Medicine), a Organização Mundial da Saúde (OMS) designa de Medicina Tradicional e Complementar: um conjunto heterogêneo de práticas, saberes e produtos agrupados por não pertencerem ao escopo da medicina convencional (outside of mainstream conventional medicine). Há políticas nacionais a respeito estabelecidas em 69 Estados Membros, com desafios para o seu desenvolvimento, dentre os quais destacamos a integração, principalmente na atenção primária à saúde 1 . Nesse sentido, é importante analisar estratégias e experiências existentes, como subsídio ao debate sobre o acesso à Medicina Tradicional e Complementar e sua inserção nos sistemas públicos de saúde.

No Brasil, há registro da Medicina Tradicional e Complementar no Sistema Único de Saúde (SUS) desde a década de 1980 4. Sua inserção no SUS foi intensificada após a Política Nacional de Práticas Integrativas e Complementares (PNPIC). Essa legitimou expressamente a oferta pública de fitoterapia, homeopatia, medicina tradicional chinesa/acupuntura e medicina antroposófica 5 , mas impulsionou também o crescimento de outras práticas que continuam a crescer ${ }^{6}$. As práticas da Medicina Tradicional e Complementar que poderiam ser consideradas tradicionais no Brasil (com exceção da fitoterapia), práticas religiosas e de curadores tradicionais, como rezadores e xamãs, por exemplo, não foram incorporadas expressamente na PNPIC, apesar de existirem em alguns municípios.

De modo geral, sua implantação no SUS tem tido pouco apoio, considerando o baixo incentivo financeiro, poucos investimentos em formação e baixa avaliação e monitoramento, sobretudo quanto à inserção da Medicina Tradicional e Complementar na atenção primária à saúde. Entretanto, existem experiências que contemplam diferentes racionalidades médicas e práticas integrativas e complementares 5,6. Segundo o Ministério da Saúde, em 2008, 25\% dos municípios brasileiros tinham oferta da Medicina Tradicional e Complementar 5, diferenciada conforme os contextos locais 6, com forte presença na atenção primária à saúde, principalmente na Estratégia Saúde da Família (ESF) 5 . As experiências municipais têm sido fruto de arranjos locais 4,6,7 e gerado um cenário diversificado de inserção da Medicina Tradicional e Complementar no SUS. Pouco se sabe acerca de como esses arranjos vêm se desenvolvendo, quais as suas possibilidades de expansão e de integração da Medicina Tradicional e Complementar com a atenção primária à saúde, bem como de que modo podem contribuir para o fortalecimento da atenção primária à saúde.

O Brasil adotou a atenção primária à saúde ou atenção básica como política pública, estruturada pela ESF 8, e é relevante visualizar como a Medicina Tradicional e Complementar tem se inserido e se integrado à mesma e aos demais serviços do SUS. Neste trabalho, o termo inserção designa a presença institucional da Medicina Tradicional e Complementar no SUS, e integração na atenção primária à saúde significa sua articulação e interação com o cuidado biomédico, compondo o leque de possibilidades terapêuticas na ESF.

Assim, este estudo objetivou analisar a inserção da medicina tradicional e complementar no SUS, tendo como foco subsidiar a discussão sobre sua integração na atenção primária à saúde, via ESF.

\section{Método}

Trata-se de um estudo qualitativo de experiências municipais baseado em estudos de casos ${ }^{9}$ previamente realizados e pesquisas em dados primários e secundários. Para seleção dos municípios, foram analisados os relatórios do Ministério da Saúde, consultas a sítios oficiais das secretarias municipais de saúde e revisão bibliográfica de experiências publicadas em revistas indexadas. Os critérios para seleção foram: presença da Medicina Tradicional e Complementar em serviços públicos por no mínimo cinco anos; presença de uma coordenação municipal ou função gestora da Medicina Tradicional e Complementar; disponibilidade de informações sobre a institucionalização da Medicina Tradicional e Complementar; heterogeneidade dos municípios entre si e similaridade quanto à oferta da Medicina Tradicional e Complementar com outros municípios, segundo o Cadastro Nacional de Estabelecimentos de Saúde (CNES) e, por fim, a conveniência da disponibilidade de dados primários 
e secundários de estudos de caso previamente realizados em outras pesquisas (Tabela 1). Os selecionados foram: Campinas (São Paulo), Florianópolis (Santa Catarina), Recife (Pernambuco), Rio de Janeiro e São Paulo.

A coleta de dados no CNES, nos sites institucionais e em bancos de dados de pesquisas anteriores, conforme o Tabela 1, ocorreu entre junho de 2014 e maio de 2015. Alguns dados foram atualizados por meio de correio eletrônico com os respectivos municípios após esse período.

A análise foi realizada em duas perspectivas: (1) inserção da Medicina Tradicional e Complementar na rede assistencial do SUS; (2) integração com a ESF/atenção primária à saúde. As seguintes dimensões de análise foram construídas a posteriori e orientaram a apresentação dos resultados: (a) inserção na agenda da saúde; (b) posição da Medicina Tradicional e Complementar na rede de serviços; (c) forma e regulação do acesso à Medicina Tradicional e Complementar; (d) profissionais praticantes e sua formação; (e) tipos de práticas/serviços oferecidos; (f) perfil da demanda; (g) potencial de

\section{Tabela 1}

Legislação da Medicina Tradicional e Complementar nos sistemas de saúde municipais, inclusão em planos plurianuais e fonte de dados dos municípios, 2015.

\begin{tabular}{|c|c|c|c|c|}
\hline Municípios & Legislação & Plano plurianual & Sites de busca dos municípios & $\begin{array}{l}\text { Dados primários do banco de } \\
\text { dados de estudo de caso }\end{array}$ \\
\hline Campinas & Não encontrado & $\begin{array}{l}\text { Faz referência à } \\
\text { política de práticas } \\
\text { integrativas. Não } \\
\text { prevê metas e } \\
\text { objetivos }\end{array}$ & http://2009.campinas.sp.gov.br/saude/ & $\begin{array}{l}\text { Entrevista com gestor e } \\
\text { profissionais em Medicina } \\
\text { Tradicional Complementar, } \\
\text { entrevista com gestor da atenção } \\
\text { primária à saúde. }\end{array}$ \\
\hline Florianópolis & $\begin{array}{c}\text { Portaria no 047/2010 } \\
\text { Portaria SS/GAB no 010/2010 }\end{array}$ & $\begin{array}{l}\text { Não faz referência à } \\
\text { política de práticas } \\
\text { integrativas }\end{array}$ & $\begin{array}{c}\text { http://www.pmf.sc.gov.br/arquivos/ } \\
\text { arquivos/pdf/28_03_2011_17.11.15.09e3 } \\
\text { 252eee513041fb6dd3d5e03189f7.pdf } \\
\text { http://www.pmf.sc.gov.br/arquivos/ } \\
\text { documentos/pdf/08_08_2014_9.04.33. } \\
\text { e92b012366d98083589 } \\
\text { e4d546c7691d1.pdf }\end{array}$ & $\begin{array}{l}\text { Entrevista com gestores } \\
\text { da Medicina Tradicional } \\
\text { Complementar e da atenção } \\
\text { primária à saúde. Entrevista com } \\
\text { profissionais da atenção primária } \\
\text { à saúde praticantes de Medicina } \\
\text { Tradicional Complementar. }\end{array}$ \\
\hline Recife & Portaria no 122/2012 & Define metas & $\begin{array}{c}\text { http://picsne.com.br/html/PMPICS } \\
\text { http://www2.recife.pe.gov.br/ } \\
\text { servico/plano-municipal-de-saude- } \\
\text { pms-2014-2017 }\end{array}$ & $\begin{array}{l}\text { Entrevista com gestores } \\
\text { da Medicina Tradicional } \\
\text { Complementar e da atenção } \\
\text { primária à saúde. Entrevista com } \\
\text { grupo focal com profissionais } \\
\text { em Medicina Tradicional } \\
\text { Complementar. }\end{array}$ \\
\hline $\begin{array}{l}\text { Rio de } \\
\text { Janeiro }\end{array}$ & $\begin{array}{c}\text { Lei no } 1.162 / 1987 \\
\text { Decreto no } 10.074 / 1989 \\
\text { Lei no } 2.899 / 1999\end{array}$ & Define objetivo & $\begin{array}{c}\text { http://mail.camara.rj.gov.br/APL/ } \\
\text { Legislativos/contlei.nsf/7cb7d306c2b74 } \\
\text { 8cb0325796000610ad8/8bcfd1d0112a5 } \\
\text { 666032576ac007388e1?OpenDocument } \\
\text { http://www.rio.rj.gov.br/ } \\
\text { dlstatic/10112/3700816/4128745/ } \\
\text { PMS_20142017.pdf }\end{array}$ & $\begin{array}{c}\text { Entrevista com gestores } \\
\text { da Medicina Tradicional } \\
\text { Complementar. Entrevista } \\
\text { com profissionais em Medicina } \\
\text { Tradicional Complementar. }\end{array}$ \\
\hline São Paulo & $\begin{array}{c}\text { Lei no } 13.717 / 2004 \\
\text { Lei no } 14.682 / 2008 \\
\text { Decreto no 49.596/2008 } \\
\text { Lei no } 14.903 / 2009\end{array}$ & $\begin{array}{l}\text { Define metas e } \\
\text { objetivos }\end{array}$ & $\begin{array}{c}\text { http://www3.prefeitura.sp.gov.br/ } \\
\text { cadlem/secretarias/negocios_juridicos/ } \\
\text { cadlem/integra.asp?alt=09012004L\%20 } \\
\text { 137170000 } \\
\text { http://prefeitura.sp.gov.br/cidade/ } \\
\text { secretarias/upload/saude/Plano_ } \\
\text { Municipal_de_Saude_Anexo.pdf }\end{array}$ & $\begin{array}{c}\text { Entrevista com profissionais } \\
\text { em Medicina Tradicional } \\
\text { Complementar. }\end{array}$ \\
\hline
\end{tabular}


expansão no SUS. A integração na atenção primária à saúde foi avaliada pela conjunção do acesso com o processo de trabalho, indicada pela articulação das dimensões "b”, "c", "d", "f”.

O foco nos modos de inserção/integração da Medicina Tradicional e Complementar e a perspectiva de contribuir para a orientação e otimização desse processo no país resultaram em uma análise comparativa da qual emergiram quatro tipos básicos de inserção da Medicina Tradicional e Complementar no SUS. Isso implicou um tratamento dos dados no sentido da caracterização e generalização dos tipos identificados, e afastou a apresentação das singularidades de cada experiência.

\section{Resultados e discussão}

Identificou-se em todos os municípios que a Medicina Tradicional e Complementar pode ter inserção em dois ou mais pontos da rede, por exemplo: ESF, unidade tradicional de atenção primária à saúde, ambulatório especializado, serviço especializado em Medicina Tradicional e Complementar, equipes de apoio à saúde da família e/ou hospital. Segundo o CNES, os municípios apresentam cenário similar aos dados nacionais, com maior oferta da Medicina Tradicional e Complementar na atenção primária (67\%). Dos estabelecimentos que dispõem do serviço de Medicina Tradicional e Complementar, 54\% são centros de saúde ou unidades básicas de saúde.

Os dados dos sistemas de informações referentes aos municípios demonstram que a maior oferta em Medicina Tradicional e Complementar é realizada pelo SUS, sendo em maior número as práticas corporais, seguidas de outras práticas em medicina chinesa. Florianópolis tem a maior e Recife a menor oferta de atendimento SUS em Medicina Tradicional e Complementar, respectivamente, 35,6 e 4,2 serviços por 100 mil habitantes (Tabela 2). Os dados acerca da oferta são similares aos de outro estudo realizado no país ${ }^{6}$. As práticas da Medicina Tradicional e Complementar em cada município são variadas e excedem a PNPIC (Tabela 3), a discussão de quais práticas podem ou devem ser contempladas, ou consideradas Medicina Tradicional e Complementar, foge do nosso objetivo.

Há uma lacuna nas políticas dos municípios quanto ao papel da Medicina Tradicional e Complementar na rede, além da ausência de uma agenda institucional e financiamento que garantam maior sustentabilidade, tal como dotação orçamentária específica. Ao analisar os Planos Plurianuais de 2014 a 2017, apenas São Paulo e Recife definiram metas para a Medicina Tradicional e Complementar (Tabela 1). Isso é corroborado pela observação dos planos municipais atuais, em que parte deles não construíram metas para a Medicina Tradicional e Complementar ou as institucionalizaram formalmente (Tabela 1).

As experiências foram desenvolvidas de maneira distinta: implantação de um serviço exclusivo, como ocorreu no Recife 10; criação de uma Política Municipal no Rio de Janeiro, após experiência em um hospital e posterior inserção na atenção básica 11; presença inicial em um centro de saúde (fitoterapia) e em ambulatório especializado (homeopatia), com posterior disseminação na atenção primária à saúde com base em práticas corporais específicas, apoiada pela gestão em Campinas 7; expansão na ESF por iniciativa da gestão, em Florianópolis 12; que têm no protagonismo dos profissionais sua maior similaridade, como em outros municípios 13,14. Nesse contexto heterogêneo, foi possível identificar quatro tipos de inserção da Medicina Tradicional e Complementar no SUS, que comumente aparecem combinados ou sozinhos.

\section{Tipo 1: inserção da Medicina Tradicional e Complementar na atenção primária à saúde via equipes de saúde da família}

Neste tipo de inserção, a Medicina Tradicional e Complementar é praticada pelos mesmos profissionais que realizam o cuidado geral dos usuários nas equipes de saúde da família. Esses profissionais são híbridos, praticantes ao mesmo tempo de alguma Medicina Tradicional e Complementar e do cuidado biomédico, em contraste com uma prática profissional exclusiva de Medicina Tradicional e Complementar ou da biomedicina. A oferta da Medicina Tradicional e Complementar é dependente da competência prévia dos profissionais ou da formação dos mesmos em serviço. No primeiro caso, a Medicina Tradicional e Complementar está presente onde os profissionais tenham obtido expertise nesta prática por conta própria. Porém, essa presença depende de iniciativas individuais e mantém 


\section{Tabela 2}

Número de serviços em Medicina Tradicional e Complementar com atendimento SUS (Sistema Único de Saúde) e não SUS e oferta por 100 mil habitantes nos municípios, 2015.

\begin{tabular}{|c|c|c|c|c|c|c|c|c|c|c|}
\hline & \multicolumn{2}{|c|}{ Campinas } & \multicolumn{2}{|c|}{ Florianópolis } & \multicolumn{2}{|c|}{ Recife } & \multicolumn{2}{|c|}{ Rio de Janeiro } & \multicolumn{2}{|c|}{ São Paulo } \\
\hline & Não & SUS & Não & SUS & Não & SUS & Não & SUS & Não & SUS \\
\hline & sus & & SUS & & SUs & & SUS & & SUS & \\
\hline Acupuntura & 6 & 19 & 12 & 19 & 7 & 8 & 4 & 22 & 49 & 78 \\
\hline Fitoterapia & 0 & 1 & 1 & 10 & 0 & 1 & 0 & 13 & 0 & 1 \\
\hline Homeopatia & 1 & 6 & 2 & 8 & 5 & 1 & 7 & 25 & 9 & 7 \\
\hline Práticas corporais & 4 & 67 & 4 & 57 & 0 & 49 & 14 & 174 & 3 & 435 \\
\hline Outras técnicas em Medicina Chinesa * & 2 & 16 & 2 & 56 & 0 & 5 & 1 & 38 & 4 & 152 \\
\hline Total & 13 & 109 & 21 & 150 & 12 & 64 & 26 & 272 & 65 & 673 \\
\hline \multicolumn{11}{|l|}{ Medicina Tradicional e Complementar } \\
\hline por 100 mil habitantes ** & 1,2 & 10,1 & 5,0 & 35,6 & 0,8 & 4,2 & 0,4 & 4,3 & 0,6 & 6,0 \\
\hline
\end{tabular}

Fonte: Departamento de Informática do SUS (Cadastro Nacional de Estabelecimentos de Saúde, 2014. http://cnes.data sus.gov.br/).

* Sessões de acupuntura com aplicação de ventosas e moxa, sessão de acupuntura com inserção de agulhas, sessão de eletroestimulação;

** Instituto Brasileiro de Geografia e Estatística (Censo Demográfico 2010. http://www.ibge.gov.br/home/estatistica/ populacao/censo2010/).

Tabela 3

Modalidades de Medicina Tradicional e Complementar oferecidas no Sistema Único de Saúde (SUS) pelos municípios, 2015.

\begin{tabular}{|c|c|c|c|c|c|}
\hline & Rio de Janeiro & Recife & Campinas & São Paulo & Florianópolis \\
\hline Homeopatia - atendimento & $X *$ & $X * *$ & $X * *$ & $X$ * & $X$ * \\
\hline Homeopatia - dispensação & $X *$ & $X * *$ & - & - & - \\
\hline Acupuntura & $X *$ & $X * *$ & $X *, * \star$ & $X *$ & $X *$ \\
\hline Massagem da Medicina Tradicional Complementar & $X *$ & - & - & $x$ & $X *$ \\
\hline Meditação (inclui da Medicina Tradicional & $x *$ & $X * \star$ & $x$ & $x$ & $X *$ \\
\hline \multicolumn{6}{|l|}{ Complementar) } \\
\hline $\begin{array}{l}\text { Práticas corporais orientais (Tai chi, Yoga, Liang long, } \\
\text { qi gong, xiang gong, Lien ch'i) }\end{array}$ & $x *$ & $X \star \star *$ & 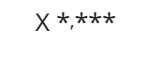 & $x$ & $X *$ \\
\hline Fitoterapia & $X *$ & $X *, \star \star$ & $x *$ & $x$ & $X *$ \\
\hline Medicina antroposófica & - & - & - & - & - \\
\hline Auriculoterapia com semente & $X *$ & $X \star \star \star \star, \star \star \star *$ & $x *$ & - & $X *$ \\
\hline Shantala & $x *$ & - & - & $x$ & - \\
\hline Reflexologia podal (inclui Tui Ná) & $x *$ & $X * *$ & - & & - \\
\hline Do in/Shiatsu/Automassagem & $x$ * & - & $x$ * & $x$ & $X *$ \\
\hline Massagem sueca & $X$ * & - & - & - & - \\
\hline Terapia comunitária & - & $X *, * \star$ & $x *$ & - & - \\
\hline Osteopatia & - & $X * *$ & $X * \star$ & - & - \\
\hline Movimento vital expressivo & - & $X * \star$ & $x *$ & - & - \\
\hline Consciência postural & - & $X * \star$ & $x$ & - & \\
\hline Microssistema de Yamamoto e Su Jok (acupuntura) & - & - & $x$ * & - & - \\
\hline Culinária integrativa/Alimentação viva & - & 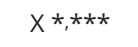 & - & $x$ & $x$ \\
\hline Danças circulares sagradas/Dança sênior & - & - & & $x$ & - \\
\hline Caminhada/Capoeira & - & $X * \star$ & $X *$ & $x$ & - \\
\hline
\end{tabular}

-: sem informação.

* Realizada em serviços de atenção primária à saúde, acesso não especificado;

** Realizada em serviço especializado, acesso por encaminhamento;

*** Realizada em serviço especializado, com acesso direto. 
pouca disseminação. Esse tipo de inserção é comum nos serviços que oferecem Medicina Tradicional e Complementar na atenção primária á saúde (com ou sem ESF) dos municípios estudados. Está ligado às primeiras experiências de oferta da Medicina Tradicional e Complementar no SUS e deriva do comum protagonismo dos profissionais praticantes ${ }^{14}$, conforme encontrado por Salles \& Schraiber 13 , e Sousa \& Vieira 11 para o caso da homeopatia.

$\mathrm{Na}$ formação proporcionada pela gestão via educação em serviço, a Medicina Tradicional e Complementar é determinada pelas iniciativas institucionais, as quais necessariamente são restritas, dado que o profissional precisa manter-se na atividade profissional enquanto realiza a formação no horário de trabalho. A formação e a expertise resultantes tendem a ser pequenas, a não ser que se proporcione cursos com aprofundamento ou de especialização no ambiente institucional. Isso tem sido raro, mas possível: encontramos a experiência de São Paulo, que em 2010 proporcionou formação para médicos da rede municipal e da saúde da família em um curso de especialização em homeopatia com ênfase em Saúde Pública e ESF 15. Outra iniciativa ocorre em Florianópolis, por meio de cursos introdutórios oferecidos aos profissionais da ESF em plantas medicinais, acupuntura, auriculoterapia, Yoga, práticas corporais e automassagem chinesa, entre outras, em parceria com a universidade e profissionais da Medicina Tradicional e Complementar do município 12.

No país é incipiente a formação em Medicina Tradicional e Complementar para o SUS, apesar de iniciativas restritas, como o curso nacional a distância de Gestão em Práticas Integrativas e Complementares e outros 16. A maior parte da formação em Medicina Tradicional e Complementar é realizada por instituições privadas que não mantêm diálogo com o sistema público de saúde ${ }^{17}$. A formação no campo da Medicina Tradicional e Complementar é um ponto crítico também em outros sistemas de saúde 18 e demanda tratamento fora do escopo deste artigo.

Há negociações locais para o exercício da Medicina Tradicional e Complementar pelos profissionais 7, com três modos comuns de operacionalização no processo de trabalho da ESF/atenção primária à saúde. $\mathrm{O}$ primeiro é a prática diluída em meio ao cuidado biomédico, sem necessitar de setting próprio (local e horário específicos) para a sua realização. O segundo modo ocorre quando há um espaço (horário) reservado na agenda dos profissionais para o exercício de alguma Medicina Tradicional e Complementar: é agendado um retorno para a prática da Medicina Tradicional e Complementar ou continuidade dela em um usuário inicialmente atendido pelo mesmo profissional no contexto biomédico. O terceiro é um misto dos dois anteriores. Dependendo do modo de operacionalização, o acesso dos usuários é diferente: quando a Medicina Tradicional e Complementar está diluída, o acesso é simultâneo e igual ao acesso ao cuidado biomédico, e a oferta do uso da Medicina Tradicional e Complementar é dependente da iniciativa dos profissionais e usuários, em comum acordo. No segundo modo, a oferta da Medicina Tradicional e Complementar continua sendo simultânea com os outros cuidados, mas a realização ou pelo menos a continuidade do tratamento, quando houver, é agendada para o horário específico da Medicina Tradicional e Complementar. Nesse caso, pode haver algum gap entre oferta e/ou início do tratamento e a sua continuidade, pois pode existir fila de espera para a Medicina Tradicional e Complementar, a depender do modo de agendamento dos serviços e da intensidade do seu uso. No terceiro caso, o acesso é uma mistura entre os dois anteriores. Os municípios onde foram observados esses três modos de operacionalização foram Campinas, Florianópolis e São Paulo.

Quanto ao perfil da demanda, este é similar ao dos atendimentos da ESF, com um grande número de casos inespecíficos, adoecimentos iniciais, quadros mal enquadráveis, sofrimentos pouco explicados. Além disso, são comuns problemas psicossociais ou de saúde mental, osteomusculares, dores em geral e doenças crônicas, para os quais várias Medicina Tradicionais e Complementares parecem ser potencialmente efetivas 1,19,20. Esse perfil de demanda para Medicina Tradicional e Complementar foi encontrado em estudos internacionais, e indica que dentro e fora dos sistemas públicos de saúde 19,20,21 parece haver um perfil específico para as mesmas.

Nesse tipo de inserção, a Medicina Tradicional e Complementar está integrada à ESF/atenção primária à saúde. Compartilha a mesma demanda da unidade de saúde, com maior ou menor exercício, conforme a intensidade do uso pelo profissional e a organização de sua equipe. Todavia, como são poucos os profissionais com expertise em Medicina Tradicional e Complementar nos serviços de ESF/atenção primária à saúde, o acesso à Medicina Tradicional e Complementar costuma ser restrito. Tendo em vista que 56,2\% dos brasileiros moram em domicílios cadastrados na ESF 22 , que é subdimensionada, o volume da demanda por assistência à saúde, de regra, é muito grande devido 
ao número excessivo de usuários vinculados às equipes. A maior pressão é pela assistência biomédica e costuma haver pouco volume proporcional de prática da Medicina Tradicional e Complementar. Dados da mais recente Pesquisa Nacional de Saúde, evidencia que o uso de práticas integrativas ocorre por 3,8\% da população (7,6 milhões de brasileiros) 22 . Há necessidade de investigações que elucidem a avaliação dos usuários e profissionais na ESF acerca desse tipo de inserção. Em outros países, médicos de família e comunidade relatam que a presença da Medicina Tradicional e Complementar na atenção primária tem trazido uma contribuição substancial 23, e outros referem interesse na integração da Medicina Tradicional e Complementar com a atenção primária à saúde 24.

\section{Tipo 2: inserção da Medicina Tradicional e Complementar na atenção primária à saúde com profissionais de exercício exclusivo}

A Medicina Tradicional e Complementar também é praticada nos serviços de atenção primária à saúde, principalmente unidades básicas tradicionais, por profissionais que em geral exercem exclusivamente Medicina Tradicional e Complementar (não operam o cuidado biomédico). $\mathrm{O}$ acesso se dá de duas maneiras: por encaminhamento de outro profissional, funcionando o praticante como referência especializada na Medicina Tradicional e Complementar exercida, e/ou por demanda espontânea direta. A organização da agenda desses profissionais é voltada para seus atendimentos com Medicina Tradicional e Complementar, conforme as peculiaridades de cada uma. Os profissionais têm inserção cotidiana no serviço, o que lhes dá maior aproximação com o universo cultural e psicossocial dos usuários, em relação aos que atendem em unidades especializadas.

O perfil da demanda atendida depende do tipo de Medicina Tradicional e Complementar e da existência ou não de protocolos e/ou prioridades estabelecidas formalmente ou não, o que, se houver, tende a gerar diferenças da demanda geral da atenção primária à saúde (dores para acupuntura, problemas alérgicos e de saúde mental para homeopatia, por exemplo). Mesmo sem protocolos rígidos, a maior parte da demanda para Medicina Tradicional e Complementar costuma ser definida pela seleção de outros profissionais biomédicos generalistas ou especializados, e isto faz com que haja diferenciação e seleção de demanda em relação ao serviço de atenção primária à saúde ou à equipe de saúde da família, como encontrado no Rio de Janeiro.

A integração da Medicina Tradicional e Complementar com a atenção primária à saúde nesse caso é bem menor, e a Medicina Tradicional e Complementar pode ser considerada justaposta a esta. Apesar de ser exercida em uma unidade de atenção primária à saúde ou ESF, isto não significa que o profissional ou a oferta da Medicina Tradicional e Complementar esteja integrada ao processo de trabalho da atenção primária à saúde. Ao contrário, há tendência de se constituir um ambulatório especializado em Medicina Tradicional e Complementar dentro de um serviço de atenção primária à saúde. É o caso dos profissionais de práticas corporais, além de outras, presentes em São Paulo e Campinas, que funcionam como referência. São Paulo conta com 1.200 profissionais em quase 500 unidades de saúde 25. Campinas dispõe de 13 polos de difusão das práticas corporais que podem ou não estar ligadas a uma unidade básica.

Também nesse caso, repete-se o problema de acesso e de pouca difusão da Medicina Tradicional e Complementar mencionada no Tipo 1: como tais profissionais são poucos no SUS e raras as iniciativas de gestores municipais de os alocar em serviços de atenção primária à saúde, a presença da Medicina Tradicional e Complementar permanece restrita e dificulta o acesso, pois está somente nas unidades dos praticantes exclusivos. A exceção são os profissionais de práticas corporais que tenham território como campo de atuação, atendendo em mais de uma unidade de saúde, como é o caso de São Paulo, Campinas e Recife. Provavelmente, não é por acaso que as práticas corporais constituem a oferta de Medicina Tradicional e Complementar que mais tem crescido no SUS 6, pela maior facilidade de sua expansão, tanto pela diversidade de profissionais que podem executá-la como pela maior disponibilidade de espaço para a sua execução (praças, academias da cidade, centros de saúde, centros comunitários etc.).

Esse tipo de inserção padece de um segundo problema: ele pressupõe o acréscimo de profissionais da Medicina Tradicional e Complementar às equipes de ESF/atenção primária à saúde, num contexto da atenção primária à saúde em que há carência de profissionais qualificados para o cuidado biomédico generalista. Ele implica que cada Medicina Tradicional e Complementar presente na ESF/atenção 
primária à saúde demanda um novo profissional, tornando insustentável a curto ou médio prazo a proposta desse arranjo como modelo para a expansão da Medicina Tradicional e Complementar na ESF/atenção primária à saúde. Outro aspecto importante é a integração desse novo profissional na atenção primária à saúde, o que demanda pensar estratégias específicas adaptadas à realidade local, como tem sido discutido em outros sistemas de saúde 26,27.

Esse tipo de inserção é relativamente comum em grandes cidades, onde é possível deslocar um profissional para a prática de uma Medicina Tradicional e Complementar numa unidade de atenção primária á saúde, e ocorre no Rio de Janeiro, São Paulo e Campinas.

Tipo 3: inserção da Medicina Tradicional e Complementar via equipes de apoio à ESF ou via Núcleo de Apoio à Saúde da Família

Neste tipo, a Medicina Tradicional e Complementar é praticada nos serviços de atenção primária à saúde por profissionais exclusivos, atuantes em equipes de apoio, por exemplo, Núcleos de Apoio à Saúde da Família (NASF) 28. Nesse caso, suas atividades muitas vezes são prioritariamente de atendimentos em grupos e de educação permanente dos profissionais da ESF, incluindo discussão de casos e matriciamento 29 em Medicina Tradicional e Complementar. Essa tem sido uma diretriz federal comumente seguida para a atuação dos NASF e equipes semelhantes, como o Núcleo de Apoio às Práticas Integrativas em Recife. A normativa federal é ambígua, pois não elimina o cuidado individual especializado referenciado. A prioridade para as atividades coletivas e o matriciamento depende dos gestores municipais e locais, que podem priorizá-los ou não. Se realizam atendimentos individuais referenciados, recaem no Tipo 2 anteriormente descrito, mas com o profissional da Medicina Tradicional e Complementar alocado na equipe de apoio ou na do NASF.

O acesso acontece comumente por encaminhamento de um profissional da atenção primária à saúde, a não ser em grupos de Medicina Tradicional e Complementar de acesso direto, o que é relativamente comum em grupos de Yoga, meditação, Liang gong, Tai chi chuan, entre outros, como em Recife. A organização da agenda desses profissionais é voltada para a Medicina Tradicional e Complementar ou dividida com outras funções de cuidado convencional, conforme as peculiaridades de cada Medicina Tradicional e Complementar e do profissional. A formação desses profissionais das equipes de apoio, como regra, é realizada previamente à sua inserção no SUS ou paralela a esta inserção. Atuam com Medicina Tradicional e Complementar exclusivamente se forem de equipes especializadas neste tipo de Medicina (Recife), ou como profissionais convencionais praticantes de alguma Medicina Tradicional e Complementar nos NASF, se não forem médicos acupunturistas e homeopatas, únicos profissionais exclusivos da Medicina Tradicional e Complementar previstos na regulamentação federal dos NASF 28 (embora os municípios possam acrescentar outros profissionais por conta própria). Esse tipo de inserção apresenta também o problema da multiplicação de profissionais nas equipes de apoio ao NASF para cada Medicina Tradicional e Complementar inserida, se forem exclusivos.

Nas equipes de apoio, há também uma maior aproximação dos profissionais com a realidade dos usuários e das ESF. O perfil da demanda atendida é similar ao do Tipo 2, e dependerá do tipo de Medicina Tradicional e Complementar e da existência ou não de protocolos e prioridades. A integração com a atenção primária é parcial, pois o profissional da Medicina Tradicional e Complementar funciona como retaguarda especializada e matriciadora.

O potencial de expansão da Medicina Tradicional e Complementar no SUS desse tipo de inserção é maior do que no Tipo 2, tendo em vista que como referências matriciadoras os praticantes da Medicina Tradicional e Complementar podem ser retaguarda de diferentes equipes da saúde da família. Esse tipo viabiliza cuidado em práticas coletivas e atenção individual aos usuários referenciados, e simultaneamente propicia contato e formação inicial, via educação permanente, aos profissionais generalistas da ESF/atenção primária à saúde. Isso permite a proliferação na ESF de atividades grupais com a Medicina Tradicional e Complementar e maior diálogo com outros profissionais desta Estratégia, diferentemente do Tipo 2, em cujo processo de trabalho o diálogo com os profissionais da ESF não é crucial e de regra é restrito a uma unidade. Além disso, as atividades coletivas ou individuais em Medicina Tradicional e Complementar são viabilizadas sem consumir tempo dos profissionais da ESF, os quais comumente estão sobrecarregados com o excesso de demanda. 
Todavia, um problema desse tipo de inserção como modelo a ser expandido é que ele é limitado no que diz respeito ao atendimento individual com a Medicina Tradicional e Complementar. Por funcionar como um serviço referenciado, implica precariedade de acesso ao cuidado individual com a Medicina Tradicional e Complementar. Isso ocorre também porque quando o profissional do NASF investe no potencial de promoção e difusão das atividades coletivas em Medicina Tradicional e Complementar, tem grande parte da sua carga horária voltada a estas atividades, restringindo o volume de atendimentos individuais.

Paradoxalmente, esse é um modelo estratégico para a implantação e expansão de retaguarda especializada em Medicina Tradicional e Complementar, pelas virtudes de contato assíduo, presencial e personalizado com os profissionais da ESF que referenciam. Assim, possibilita discussão de casos, regulação e educação permanente. Entretanto, se os profissionais dos NASF fossem vistos como referência especializada para atendimentos individuais, estes profissionais teriam menos tempo para as atividades coletivas, incluindo em Medicina Tradicional e Complementar. Essa tensão necessita ser trabalhada localmente de alguma forma, mas ela atenuaria com a proliferação dessas equipes. Mesmo assim, nesse tipo de inserção, o cuidado individual com Medicina Tradicional e Complementar permanece referenciado, o que significa restrito a casos mais graves ou complexos.

Esse tipo de inserção é comum onde há NASF ou equipes de apoio à ESF/atenção primária à saúde com atuação em Medicina Tradicional e Complementar, e foi encontrado em Recife.

\section{Tipo 4: inserção da Medicina Tradicional e Complementar em unidades especializadas}

Neste tipo, a Medicina Tradicional e Complementar é exercida em unidades especializadas biomédicas ou de Medicina Tradicional e Complementar por praticantes exclusivos deste tipo de Medicina, que podem não ser das profissões da saúde, uma vez que o regime de vinculação pode ser por contrato. Em princípio, a formação e a prática especializada em tempo integral propicia maior expertise, o que pode ser útil em casos mais graves ou clinicamente complexos. $\mathrm{O}$ acesso se dá geralmente por encaminhamento de outro profissional da ESF/atenção primária à saúde ou especialista, ou por demanda espontânea conforme as regras locais, em alguns serviços que oferecem exclusivamente a Medicina Tradicional e Complementar. A organização da agenda desses profissionais é voltada para seus atendimentos com Medicina Tradicional e Complementar. É comum haver longas filas e demora para o início do atendimento, em função do reduzido número desses profissionais, bem como da oferta desses serviços 30. Esse tipo foi observado em São Paulo, Campinas, Florianópolis (em ambulatórios especializados) e Recife (onde há duas unidades especializadas apenas em Medicina Tradicional e Complementar).

Os profissionais trabalham em serviços de referência em Medicina Tradicional e Complementar ou em serviços especializados biomédicos com Medicina Tradicional e Complementar, comumente acupuntura e/ou homeopatia. Nesse modo de inserção, o perfil da demanda depende do tipo de Medicina Tradicional e Complementar e dos encaminhamentos realizados que podem ou não ter protocolos ou diretrizes de priorização para referência. Geralmente a vinculação dos usuários a essas unidades e a comum cronicidade dos problemas dificultam a alta 31,32 . A oferta nas unidades especializadas em Medicina Tradicional e Complementar tende a ser mais diversificada. Todavia, sua inserção no SUS, pelos serviços diferenciados oferecidos em Medicina Tradicional e Complementar, pode não ser identificada como uma unidade especializada comum, e não entrar no fluxo de referência e contrarreferência da ESF/atenção primária à saúde.

Esse tipo de inserção de Medicina Tradicional e Complementar no SUS apresenta problemas semelhantes aos Tipos 2 e 3 quanto ao acesso e à sustentabilidade institucional da expansão de profissionais exclusivos de Medicina Tradicional e Complementar. Outro problema é que nele os usuários, em geral, chegam tardiamente, com menos chances de obterem benefício do que se estivessem em situações menos graves - por hipótese, coerente com o tipo de atuação da maior parte das Medicina Tradicional e Complementar, notadamente as vitalistas, que agem sobre o potencial de autocura dos indivíduos 33. Por outro lado, a gravidade dos casos, que merecem cuidados biomédicos especializados, sugere também esforços especializados em Meidicina Tradicional e Complementar, usada comumente de forma complementar, e muito procurada pelos efeitos adversos da biomedicina 19 . 
A presença da Medicina Tradicional e Complementar em unidades especializadas vem crescendo, principalmente para doenças crônicas. O ambiente especializado parece ser o que mais produz evidências científicas sobre a Medicina Tradicional e Complementar e há significativo uso e pesquisa em adoecimentos graves, como na oncologia 32,34, o que aponta para a necessidade de discutir o seu papel nos serviços especializados e hospitalares. No entanto, seu potencial de expansão parece limitado dada a necessidade de organização e expansão dos serviços hospitalares no SUS, em que profissionais biomédicos têm prioridade em relação à Medicina Tradicional e Complementar. Quanto à atenção especializada ambulatorial, este tipo de inserção também é limitado e tem os problemas de distanciamento da atenção primária à saúde, sendo que o Tipo 3 mostra-se evidentemente mais integrado devido ao matriciamento. As características de cada tipo de inserção da Medicina Tradicional e Complementar e sua integração com a atenção primária à saúde estão sintetizadas na Tabela 4.

\section{Expansão da Medicina Tradicional e Complementar no SUS e sua integração com a atenção primária à saúde}

A inserção da Medicina Tradicional e Complementar no SUS, em uma perspectiva de ampliação da sua oferta, não é plenamente contemplada em nenhum desses modos de inserção isoladamente. Conforme sua crescente valorização social e acadêmica, sua inserção deve ser orientada de modo adequado à realidade do SUS e às necessidades sanitárias do país, com incentivo financeiro sustentável. Coerente com isso, extraiu-se uma diretriz potencial para a inserção da Medicina Tradicional e Complementar no SUS e sua integração na atenção primária à saúde, com base na análise precedente, delineada pela combinação do Tipo 1 com o Tipo 3 de inserção.

A inserção progressiva e mais disseminada da Medicina Tradicional e Complementar na atenção primária à saúde via profissionais da ESF, por meio da educação permanente e otimização das expertises existentes, é uma via com potencial de socialização dessas práticas e de aproveitamento de suas virtudes, tanto na promoção da saúde 33 como no cuidado individual e coletivo. Isso converge

\section{Tabela 4}

Modelos de inserção da Medicina Tradicional e Complementar no Sistema Único de Saúde (SUS) e integração na atenção primária à saúde.

\begin{tabular}{|c|c|c|c|c|}
\hline Dimensões & $\begin{array}{c}\text { Tipo 1: na atenção } \\
\text { primária à saúde } \\
\text { via profissionais das } \\
\text { equipes de saúde da } \\
\text { família }\end{array}$ & $\begin{array}{l}\text { Tipo 2: na atenção } \\
\text { primária à saúde } \\
\text { via profissionais de } \\
\text { exercício exclusivo }\end{array}$ & $\begin{array}{c}\text { Tipo 3: na atenção } \\
\text { primária à saúde via } \\
\text { equipes matriciais }\end{array}$ & $\begin{array}{l}\text { Tipo 4: em serviços } \\
\text { especializados }\end{array}$ \\
\hline Regulação do acesso & Demanda espontânea & Demanda referenciada & $\begin{array}{c}\text { Demanda referenciada ou } \\
\text { livre nas ações coletivas }\end{array}$ & Demanda referenciada \\
\hline $\begin{array}{l}\text { Profissional praticante } \\
\text { da Medicina Tradicional } \\
\text { Complementar }\end{array}$ & $\begin{array}{c}\text { Profissionais da atenção } \\
\text { primária à saúde/ } \\
\text { equipe de saúde da } \\
\text { família especialistas ou } \\
\text { praticantes }\end{array}$ & Praticantes exclusivos & $\begin{array}{c}\text { Praticantes exclusivos ou } \\
\text { especialistas }\end{array}$ & $\begin{array}{c}\text { Praticantes exclusivos ou } \\
\text { especialistas }\end{array}$ \\
\hline Práticas oferecidas & $\begin{array}{l}\text { Definidas pelos } \\
\text { profissionais ou gestão }\end{array}$ & Definidas pela gestão & Definidas pela gestão & Definidas pela gestão \\
\hline Perfil da demanda & $\begin{array}{c}\text { Igual à atenção primária } \\
\text { à saúde }\end{array}$ & $\begin{array}{c}\text { Semelhante à atenção } \\
\text { primária à saúde ou } \\
\text { referenciada }\end{array}$ & Referenciada & Referenciada \\
\hline $\begin{array}{l}\text { Potencial de expansão } \\
\text { no SUS }\end{array}$ & Grande & Pequeno & Grande & Pequeno \\
\hline $\begin{array}{l}\text { Integração com a equipe } \\
\text { de saúde da família }\end{array}$ & Integrada & Justaposta & Matriciada & Sem integração \\
\hline
\end{tabular}


com a ideia de que a Medicina Tradicional e Complementar contribui para a integralidade da atenção, por meio de seus distintos saberes e valores, sobretudo das racionalidades médicas vitalistas e suas diversas técnicas heterônomas e autônomas. Seus recursos enriquecedores das experiências de cuidado podem ser úteis no manejo da excessiva biomedicalização 34 e na redução de danos 35 , sendo adequados tecnologicamente ao ambiente da ESF 36.

Assim, seu lócus ideal no SUS é a ESF, em que pode ser muito importante. Porém, não encontramos evidências científicas quantitativas no Brasil sobre essa diretriz, que converge com McWhiney 36, um clássico da medicina de família, e com os praticantes da Medicina Tradicional e Complementar na ESF/atenção primária à saúde. Na literatura internacional, um estudo holandês mostrou que usuários de médicos de família também homeopatas, acupunturistas ou antroposóficos (híbridos) tiveram menos internações e prescrição de drogas e menores custos do cuidado 37 . Outros estudos evidenciam que a Medicina Tradicional e Complementar tem sido custo-efetiva para os sistemas de saúde e seguros de saúde 38,39. Também houve diminuição nos encaminhamentos para fisioterapia e reumatologia em serviço de atenção primária à saúde em que foi introduzida uma técnica de acupuntura 40 .

A inserção da Medicina Tradicional e Complementar na atenção primária à saúde via profissionais da ESF depende de iniciativas dos gestores municipais, e provavelmente de indução federal para ser acelerada. Ela demanda um importante componente de educação continuada e permanente, para isto devem ser utilizadas as expertises dos profissionais com competência em Medicina Tradicional e Complementar que atuam na ESF/atenção primária à saúde e em outros serviços. Também cabe a oferta de formação consistente ou especializações em serviço, para diminuir a superficialidade das competências em Medicina Tradicional e Complementar que atividades educativas introdutórias conseguem gerar.

O modo promissor de inserção da Medicina Tradicional e Complementar no SUS é prioritariamente na ESF, como vem ocorrendo, via profissionais híbridos (Tipo 1). Mas é coerente e complementar que seja associada com o Tipo 3, devido a esta última incluir atividades de retaguarda especializada associadas com matriciamento, com atenuação do problema das filas de espera. Há sinergia entre ambas, que permite maior integração em rede e avança na superação da distância entre atenção primária à saúde e atenção especializada. Além disso, esses praticantes da Medicina Tradicional e Complementar nas equipes de apoio podem protagonizar as atividades educativas para os profissionais da ESF e multiplicar atividades coletivas em Medicina Tradicional e Complementar. Essa associação constitui o caminho, entre as experiências analisadas, para a expansão e exploração dos potenciais da Medicina Tradicional e Complementar no cuidado institucional, de modo a aperfeiçoar e fortalecer a atenção primária à saúde e o SUS.

Experiências internacionais de integração da Medicina Tradicional e Complementar com a atenção primária em à saúde ocorrem em locais onde esta última está mais estruturada e a Medicina Tradicional e Complementar socialmente mais regulamentada do que no Brasil, como em alguns países europeus ${ }^{41}$, ou numa situação diferente como os Estados Unidos ${ }^{42}$, que têm o sistema de saúde majoritariamente privado. Também ocorrem onde a atenção primária à saúde tem grandes desafios na sua estruturação, como em países africanos 1 , destacando o papel de benzedeiras e rezadores na assistência à saúde mental ${ }^{43}$. Assim, há pouca relação entre os contextos institucionais dessas experiências e o SUS, dificultando a sua comparação com as experiências brasileiras. Além disso, o sincretismo cultural brasileiro torna o uso da Medicina Tradicional e Complementar singular, o que requer investigações mais detalhadas dessas práticas.

O maior limite deste trabalho é ter analisado experiências de municípios com maior porte populacional (mais de 100 mil habitantes), que dispõem de mais informações públicas on-line. Desse modo, são necessárias mais pesquisas nesses e em outros municípios, sobretudo de menor porte. Também merecem investigações as experiências hospitalares com Medicina Tradicional e Complementar, aqui não tematizadas.

\section{Considerações finais}

A significativa e crescente presença da Medicina Tradicional e Complementar no SUS demanda pensar estrategicamente sua expansão, para além da PNPIC, cuja única diretriz organizativa é a genérica 
ênfase na atenção básica. A sua inserção progride de forma relativamente errática e não deveriam ser desperdiçadas as experiências existentes. Ao contrário, devem ser estudadas e valorizadas, numa perspectiva de minimização da produção de sua ausência aparente e da maximização da sua emergência 44, gerando visibilidade, aprendizado institucional e político. É estratégico se extrair delas diretrizes orientadoras da inserção da Medicina Tradicional e Complementar na ESF/atenção primária à saúde e no SUS.

\section{Colaboradores}

Os autores participaram igualmente de todas as etapas.

\section{Agradecimentos}

Ao Conselho Nacional de Desenvolvimento Científico e Tecnológico pelo financiamento (CNPq processo no 401458/2013-0 e 207603/2014-6). 


\section{Referências}

1. World Health Organization. WHO traditional medicine strategy: 2014-2023. Geneva: World Health Organization; 2013.

2. Frass M, Strassl RP, Friehs H, Müllner M, Kundi $\mathrm{M}$, Kaye AD. Use and acceptance of complementary and alternative medicine among the general population and medical personnel: a systematic review. Ochsner J 2012; 12:45-56.

3. Barnes PM, Bloom B, Nahin RL. Complementary and alternative medicine use among adults and children: United States, 2007. Natl Health Stat Report 2008; (12):1-23.

4. Justo CMP, Gomes MHA. A cidade de Santos no roteiro da expansão da homeopatia nos serviços públicos de saúde no Brasil. Hist Ciênc Saúde -Manguinhos 2007; 14:1159-71.

5. Ministério da Saúde. Práticas integrativas e complementares em saúde: uma realidade no SUS. Revista Brasileira Saúde da Família 2008; http://189.28.128.100/dab/docs/publicacoes/re vistas/revista_saude_familia18_especial.pdf (acessado em 02/Fev/2015).

6. Sousa IMC, Bodstein RCA, Tesser CD, Santos FAZ, Hortale VA. Práticas integrativas e complementares: oferta e produção de atendimentos no SUS e em municípios selecionados. Cad Saúde Pública 2012; 28:2143-54.

7. Nagai SC, Queiroz MS. Medicina complementar e alternativa na rede básica de serviços de saúde: uma aproximação qualitativa. Ciênc Saúde Coletiva $2011 ; 16: 1793-800$.

8. Paim JS, Travassos C, Almeida C, Bahia L, Macinko J. The Brazilian health system: history, advances, and challenges. Lancet 2011; 377:1778-97.

9. Pires A. Amostragem e pesquisa qualitativa: ensaio teórico e metodológico. In: Poupart J, Deslauriers J-P, Groulx L-H, Laperrière A, Mayer R, Pires AP, organizadores. A pesquisa qualitativa: enfoques epistemológicos e metodológicos. Rio de Janeiro: Editora Vozes; 2008. p. 154-211.

10. Santos FAS, Sousa IMC, Gurgel IGD, Bezerra AFB, Barros NF. Política de práticas integrativas em Recife: análise da participação dos atores. Rev Saúde Pública 2011; 45:1154-9.

11. Sousa IMC, Vieira ALS. Serviços públicos de saúde e medicina alternativa. Ciênc Saúde Coletiva 2005; 10 Suppl:255-66.

12. Santos MC, Tesser CD. Um método para a implantação e promoção de acesso às Práticas Integrativas e Complementares na Atenção Primária à Saúde. Ciênc Saúde Coletiva 2012, 17:3011-24.

13. Salles SAC, Schraiber LB. Gestores do SUS: apoio e resistências à homeopatia. Cad Saúde Pública 2009; 25:195-202.

14. Brunning MCR, Mosegui GBG, Vianna CMM. A utilização da fitoterapia e de plantas medicinais em unidades básicas de saúde nos municípios de Cascavel e Foz do Iguaçu - Paraná: a visão dos profissionais de saúde. Ciênc Saúde Coletiva 2012; 17:2675-85
15. Associação Saúde da Família. Centro de referência de homeopatia, medicinas tradicionais e práticas integrativas bosque da saúde. http://www.saude dafamilia.org/projetos/centro_referencia/centro_ referencia.htm (acessado em 10/Ago/2015).

16. Coordenação Geral de Áreas Técnicas, Departamento de Atenção Básica, Ministério da Saúde. Curso de capacitação em gestão de práticas integrativas e complementares. https://cursos.aten caobasica.org.br/courses/7803 (acessado em 20/ Ago/2015).

17. Azevedo E, Pelicioni MCF. Práticas integrativas e complementares e desafios para a educação. Trab Educ Saúde 2011; 9:361-78.

18. Chung VCH, Ma PHX, Hong LC, Griffiths SM. Organizational determinants of interprofessional collaboration in integrative health care: systematic review of qualitative studies. PLoS One 2012; 7:e50022.

19. Sirois FM. Motivations for consulting complementary and alternative medicine practitioners: a comparison of consumers from 1997-8 and 2005. BMC Complement Altern Med 2008; 8:16.

20. Gureje O, Nortje G, Makanjuola V, Oladeji B, Seedat S, Jenkins R. The role of global traditional and complementary systems of medicine in treating mental health problems. Lancet Psychiatry 2015; 2:168-77.

21. Hunt KJ, Coelho HF, Wider B, Perry R, Hung SK, Terry R, et al. Complementary and alternative medicine use in England: results from a national survey. Int J Clin Pract 2010; 64:1496.

22. Instituto Brasileiro de Geografia e Estatística. PNS 2013: três em cada quatro brasileiros costumam buscar atendimento médico na rede pública de saúde. http://saladeimprensa.ibge.gov.br/ $\mathrm{pt} /$ noticias? view $=$ noticia\&id $=1$ \&busca $=1$ \&idnoti cia $=2902$ (acessado em 02/Ago/2009).

23. Joos S, Musselmann B, Szecsenyi J. Integration of Complementary and Alternative Medicine into Family Practices in Germany: results of a National Survey. Evid Based Complement Alternat Med 2011; 2011:495813.

24. Sundberg T, Halpin J, Warenmark A, Falkenberg $\mathrm{T}$. Towards a model for integrative medicine in Swedish primary care. BMC Health Serv Res 2007; 7:107.

25. Barolho C, Cabral M. Um dos maiores programas de práticas integrativas e complementares da América Latina: São Paulo. Rev Bras Med Fam Comunidade 2012; 7 Suppl 1:6-7.

26. Mior S, Barnsley J, Boon H, Ashbury FD, Haig R. Designing a framework for the delivery of collaborative musculoskeletal care involving chiropractors and physicians in community-based primary care. J Interprof Care 2010; 24:678-89.

27. Chung VCH, Ma PHX, Lau CH, Griffiths SM. Developing policy for integrating biomedicine and traditional chinese medical practice using focus groups and the delphi technique. Evid Based Complement Alternat Med 2012; 2012:149512. 
28. Departamento de Atenção Básica, Secretaria de Atenção à Saúde, Ministério da Saúde. Diretrizes do NASF - Núcleo de Apoio à Saúde da Família. http://bvsms.saude.gov.br/bvs/publicacoes/cader no_atencao_basica_diretrizes_nasf.pdf (acessado em 21/Abr/2015).

29. Campos GWS, Domitti AC. Apoio matricial e equipe de referência: uma metodologia para gestão do trabalho interdisciplinar em saúde. Cad Saúde Pública 2007; 23:399-407.

30. Lima KMSV, Silva KL, Tesser CD. Práticas integrativas e complementares e relação com promoção da saúde: experiência de um serviço municipal de saúde. Interface Comun Saúde Educ 2014; 18:26172 .

31. Levin JS, Jonas WB. Tratado de medicina complementar e alternativa. São Paulo: Manole; 2001.

32. Siegel P, Barros NF. O que é a oncologia integrativa? Cad Saúde Colet (Rio J.) 2013; 21:348-54.

33. Tesser CD. Práticas complementares, racionalidades médicas e promoção da saúde: contribuições pouco exploradas. Cad Saúde Pública 2009; 25:1732-42.

34. Clarke AE, Mamo L, Fosket JR, Fishman JR, Shim JK. Biomedicalization: technoscience, health, and illness in the U.S. Durham: Duke University Press; 2010.

35. Tesser CD, Barros NF. Medicalização social e medicina alternativa e complementar: pluralização terapêutica do Sistema Único de Saúde. Rev Saúde Pública 2008; 42:914-20.

36. McWhinney IR. Manual de medicina de família e comunidade. 3a Ed. Porto Alegre: Editora Artmed; 2010.

37. Kooreman P, Baars EW. Patients whose GP knows complementary medicine tend to have lower costs and live longer. Eur J Health Econ 2012; 13: 769-76.
38. Baars EW, Kooreman P. A 6-year comparative economic evaluation of healthcare costs and mortality rates of Dutch patients from conventional and CAM GPs. BMJ Open 2014; 4:e005332.

39. Herman PM, Poindexter BL, Witt CM, Eisenberg DM. Are complementary therapies and integrative care cost-effective? A systematic review of economic evaluations. BMJ Open 2012; 2 pii:e001046.

40. Ross J. An audit of the impact of introducing microacupuncture into primary care. Acupunct Med 2001; 19:43-5.

41. Eardley S, Bishop FL, Prescott P, Cardini F, Brinkhaus B, Santos-Rey K, et al. A systematic literature review of complementary and alternative medicine prevalence in EU. Forsch Komplementmed 2012; 19 Suppl 2:18-28.

42. Goldstein MS. A timely opportunity: fostering new roles for complementary and alternative health care providers in meeting the Nation's need for primary health care. Explore (NY) 2013; 9: 344-7.

43. Musyimi CW, Mutiso VN, Nandoya ES, Ndetei DM. Forming a joint dialogue among faith healers, traditional healers and formal health workers in mental health in a Kenyan setting: towards common grounds. J Ethnobiol Ethnomed 2016; 12:4.

44. Santos BS. Para uma sociologia das ausências e uma sociologia das emergências. Revista Crítica de Ciências Sociais 2002; 63:237-80.Tabela 1: Legislação da Medicina Tradicional e Complementar nos sistemas de saúde municipais, inclusão em planos plurianuais e fonte de dados dos municípios, 2015. 


\section{Abstract}

This study aimed to analyze the inclusion of Traditional and Complementary Medicine in Brazilian Unified National Health System (SUS) and its integration with primary healthcare (PHC). A qualitative study drew on institutional data, indexed articles, and case studies in selected Brazilian cities: Campinas (São Paulo State), Florianópolis (Santa Catarina State), Recife (Pernambuco State), Rio de Janeiro, and São Paulo. The analysis adopted the perspective of inclusion of Traditional and Complementary Medicine in the healthcare network and its integration with primary healthcare, based on the following dimensions: presence of Traditional and Complementary Medicine on the municipal agenda; position in the services; mode of access to Traditional and Complementary Medicine; Traditional and Complementary Medicine practitioners; types of practices; demand profile; and potential for expansion in the SUS. The authors identified and characterized four types of inclusion and integration of Traditional and Complementary Medicine, whether in association or not: Type 1 - in primary healthcare via professionals from the family health teams Integrated; Type 2 - in primary healthcare via professionals with full-time employment - Juxtaposed; Type 3 - in primary healthcare via matrixorganized teams - Matrix Organization; Type 4 - in specialized services - Without Integration. The combination of types 1 and 3 was considered a potential guideline for the expansion of Traditional and Complementary Medicine in the SUS and can orient the growth and integration of Traditional and Complementary Medicine with primary healthcare. The growing presence of Traditional and Complementary Medicine in the SUS requires conceiving its strategic expansion, while existing experiences should not be wasted.

Traditional Medicine; Complementary Therapies; Primary Health Care; Unified Health System

\section{Resumen}

Este estudio tuvo como objetivo analizar la inserción de la Medicina Tradicional y Complementaria en el Sistema Único de Salud y su integración con la atención primaria en la salud. Se realizó una investigación cualitativa, basada en datos institucionales, artículos indexados y estudios de casos en municipios brasileños seleccionados: Campinas (São Paulo), Florianópolis (Santa Catarina), Recife (Pernambuco), Río de Janeiro y São Paulo. El análisis fue realizado desde la perspectiva de la inserción de la Medicina Tradicional y Complementaria en la red asistencial y su integración con la atención primaria en la salud, mediante las siguientes dimensiones: presencia de la Medicina Tradicional y Complementaria en la agenda municipal; posición en los servicios; modo de acceso a la Medicina Tradicional y Complementaria; practicantes; tipos de prácticas; perfil de la demanda; potencial de expansión en el SUS. Se identificaron $y$ caracterizaron cuatro tipos de inserción e integración de la Medicina Tradicional y Complementaria, asociados o no: Tipo 1 -en la atención primaria en la salud vía profesionales de los equipos de salud da familia-Integrada; Tipo 2 -en la atención primaria en la salud vía profesionales de ejercicio exclusivo-Justaposta; Tipo 3 -en la atención primaria en la salud vía equipos matricialesMatriciada; Tipo 4 -en servicios especializadosSin integración. La combinación de los tipos 1 y 3 fue considerada una directriz potencial para la expansión de la Medicina Tradicional y Complementaria en el SUS y puede orientar el crecimiento y su integración en la atención primaria en la salud. La creciente presencia de la Medicina Tradicional y Complementaria en el SUS demanda pensar estratégicamente su expansión, y no deben ser desperdiciadas las experiencias existentes.

Medicina Tradicional; Terapias Complementarias; Atención Primaria de Salud; Sistema Único de Salud
Recebido em 17/Set/2015

Versão final reapresentada em 26/Jan/2016

Aprovado em 06/Abr/2016 